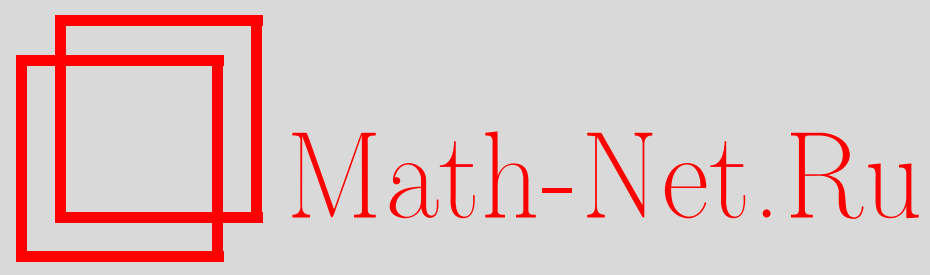

М. В. Дерябин, О полиномиальных интегралах динамических систем и редукции Лакса, Матем. заметки, 1997, том 61, выпуск 3, 445-446

DOI: https://doi.org/10.4213/mzm1519

Использование Общероссийского математического портала Math-Net.Ru подразумевает, что вы прочитали и согласны с пользовательским соглашением http://www.mathnet.ru/rus/agreement

Параметры загрузки:

IP : 54.174 .149 .18

26 апреля 2023 г., 16:10:05

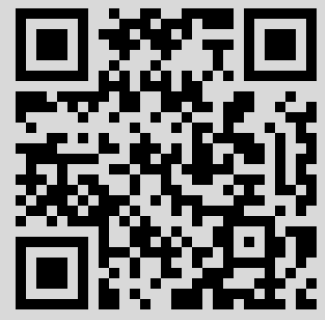




\section{О ПОЛИНОМИАЛЬНЫХ ИНТЕГРАЛАХ ДИНАМИЧЕСКИХ СИСТЕМ И РЕДУКЦИИ ЛАКСА}

\section{М.В. Дерябин}

Вопрос о существовании первых интегралов уравнений классической механики в виде полиномов по скоростям изучается уже давно. Это связано с тем, что все известные первые интегралы либо полиномиальные, либо являются функциями от полиномов. Известны условия существования полиномиальных интегралов первой и второй степени [1], [2]: все линейные интегралы нётеровы, а существование квадратичных интегралов связано с возможностью разделения переменных.

В работе [3] рассматривался вопрос о наличии полиномиального интеграла у системы с полутора степенями свободы

$$
\ddot{x}=-V_{x}
$$

где потенциал $V(x, t) 2 \pi$-периодичен по $x$. Была получена система уравнений, которой должны удовлетворять коэффищиенты полиномиального интеграла и потенциал. Оказьвается, что если первьй интеграл уравнения (1) имеет вид

$$
a_{0}(x, t)+a_{1}(x, t) \dot{x}+\cdots+a_{n}(x, t) \dot{x}^{n},
$$

где коэффициенты $a_{k}(0 \leqslant k \leqslant n) 2 \pi$-периодичны по $x$, то указанная система уравнений имеет вид

$$
\begin{gathered}
n V_{t}+\left(a_{n-3}\right)_{x}=0 \\
\left(a_{n-3}\right)_{t}+\left(a_{n-4}\right)_{x}=(n-2) n V V_{x} \\
\left(a_{n-4}\right)_{t}+\left(a_{n-5}\right)_{x}=(n-3) a_{n-3} V_{x} \\
\ldots \ldots \ldots \ldots \ldots \ldots \ldots \ldots \ldots \ldots \ldots \ldots \ldots \ldots \\
\left(a_{1}\right)_{t}+\left(a_{0}\right)_{x}=2 a_{2} V_{x} \\
\left(a_{0}\right)_{t}=a_{1} V_{x}
\end{gathered}
$$

причем без ограничения общности можно положить коэффициенты $a_{n}=1$ и $a_{n-1}=0$.

Система уравнений в частных производных (2) - это система гидродинамического типа [4]. Диагонализируемые (допускающие инварианты Римана) системы, обладающие бесконечным числом находящихся в инволюции интегралов гидродинамического типа (законов сохранения), задаваемые с произволом $n$ функций одного переменного, назьваются гидродинамически интегрируемыми. Такие системы интегрируются "обобщенным методом годографо" [5].

Исследуем вопрос о гидродинамической интегрируемости системы (2).

ПРЕДЛОЖЕНИЕ. Система уравнений (2) сводится $к$ недисперсионным уравнениям Лакса или "редукчии Лакса уравнений Бенни"

$$
u_{t}^{i}=\sum_{j=1}^{N} A_{j}^{i}(u) u_{x}^{j}, \quad i=1, \ldots, N
$$


где матрииа А равна

$$
A_{j}^{i}=\left(\begin{array}{cccccc}
0 & 1 & 0 & 0 & 0 & \ldots \\
-(N-1) u^{1} & 0 & 1 & 0 & 0 & \ldots \\
-(N-2) u^{2} & 0 & 0 & 1 & 0 & \ldots \\
\ldots \ldots \ldots \ldots \ldots \ldots \ldots & \ldots \ldots \ldots & \ldots \\
-u^{N-1} & 0 & \ldots & \ldots & 0
\end{array}\right), \quad N=n-1 .
$$

Для доказательства достаточно сделать линейную замену переменных

$$
V=u_{1}, \quad a_{n-3}=-n u_{2}, \quad a_{n-4}=n u_{3}, \ldots, \quad a_{0}= \pm n u_{n-1} .
$$

ЗАМЕчАнИЕ. При $N=1$ система (3) превращается в одно уравнение (недисперсионное уравнение Кортевега-де Фриза) [6]

$$
u_{t}=u u_{x}
$$

После замены $u \rightarrow-u$ оно переходит в известное уравнение Хопфа.

Система (3) диагонализируема: инварианты Римана ищутся из соотношения

$$
\frac{1}{n} R_{k}^{n}=\frac{1}{n} \lambda_{k}^{n}+u_{1} \lambda_{k}^{n-1}+\cdots+u_{n-1}, \quad k=1, \ldots, n-1,
$$

где $\lambda_{k}$ - собственные значения матрищы $A(4)$. При этом оказывается, что если выразить $\lambda$ через $R$ из соотношения (6)

$$
\lambda=R-\frac{H_{1}}{R}-\frac{H_{2}}{R^{2}}-\cdots,
$$

то коэффициенты $H_{1}, H_{2}, \ldots$ будут плотностями законов сохранения [6]. Таким образом, уравнения Лакса (3), а следовательно, и система уравнений (2) гидродинамически интегрируема.

Метод годографа - это локальная процедура, которая не решает вопрос о существовании решения системы (2) на всем интервале времени.

Известно, что одномерное уравнение Лакса (5) с периодичным по $x$ начальным условием не определяется однозначно на всем интервале $t$. По-видимому, из-за отсутствия дисперсии при любых значениях $N$ найдутся такие начальные условия у системы, что ее решение будет определено лишь на конечном интервале времени.

Московский государственный университет

Поступило

им. М.В. Ломоносова

17.10 .96

E-mail: derjabin@mvd.pvt.msu.su

\section{СПИСОК ЦИТИРОВАННОЙ ЛИТЕРАТУРЫ}

1. Уиттекер Е. Т. Аналитическая динамика. М.-Л.: Гостехиздат, 1937. 2. Биркгоф Дж. Динамические системы. М.-Л.: Гостехиздат, 1941. 3. Козлов В. В. // Матем. заметки. 1989. Т. 45. № 4. С. 46-52 . 4. Дубровин Б. А., Новиков С. П. // Докл. АН СССР. 1983. Т. 270. № 4. С. 781-785. 5. Царев С. П. // Докл. АН СССР. 1985. Т. 282. № 3. С. 534-537. 6. Kodama Y., Gibbons J. // Phys. Lett. A. 1989. V. 135. № 3. P. 167-170. 